PHYSICAL REVIEW D 90, 099901(E) (2014)

\title{
Erratum: Thermal field theory in a layer: Applications of thermal field theory methods to the propagation of photons in a two-dimensional electron sheet [Phys. Rev. D 81, 075007 (2010)]
}

\author{
José F. Nieves \\ (Received 29 October 2014; published 13 November 2014)
}

DOI: 10.1103/PhysRevD.90.099901

PACS numbers: 11.10.Wx, 99.10.Cd

In various equations it is not clearly indicated in some cases that the symbols representing some vector variables actually stand for their transverse counterpart. To clarify this, the replacements indicated below should be made. (The calculations and results, including the discussion of the calculation of the dispersion relations, are not affected by these).

(1) In Eq. (2.7), replace $\tilde{g}_{\mu \nu}$ by $\tilde{g}_{\perp \mu \nu}$ in the formula for $R_{\mu \nu}$, and add the following formulas:

$$
g_{\perp \mu \nu}=g_{\mu \nu}+n_{\mu} n_{\nu}, \quad \tilde{g}_{\perp \mu \nu}=\tilde{g}_{\mu \nu}+n_{\mu} n_{\nu} .
$$

(2) In Eq. (3.7), add the following formula:

$$
\bar{\psi} \gamma_{\mu} \psi=\delta(z) \overline{\hat{\psi}} \gamma_{\perp \mu} \hat{\psi}
$$

(3) On the right-hand side of Eq. (4.1), $\gamma_{\mu}$ should be replaced by $\gamma_{\perp \mu}$.

(4) In the right-hand side of Eq. (4.2), $A_{\mu}$ should be replaced by $A_{\perp \mu}$.

(5) Replace Eq. (4.3) by

$$
J^{\mu}(x)=\delta(z) \hat{J}^{\mu}\left(x_{\perp}\right)
$$

(6) In Eq. (4.4), add the formula

$$
\hat{J}\left(x_{\perp}\right) \cdot n=0 .
$$

(7) In Eq. (4.14), $\tilde{g}_{\nu}^{\mu}$ should be replaced by $\tilde{g}_{\perp \nu}^{\mu}$.

(8) In Eq. (5.1), $\gamma_{\mu}$ and $\gamma_{\nu}$ should be replaced by $\gamma_{\perp \mu}$ and $\gamma_{\perp \nu}$, respectively.

(9) In Eq. (5.4), $g_{\mu \nu}$ should be replaced by $g_{\perp \mu \nu}$.

The following typographical errors should be corrected:

(1) In Eq. (5.8), the factor $2 e^{2}$ in the formula for $\pi_{T}^{(m)}$ should be $4 e^{2}$.

(2) In Eq (5.9), the term $2 m_{e}^{2}-2 p_{\perp} \cdot k_{\perp}$ in the numerator of the integrand in the formula for $A_{e}$ should be $2 m_{e}^{2}-p_{\perp} \cdot k_{\perp}$ (i.e., without the factor of 2 in $p_{\perp} \cdot k_{\perp}$ ).

(3) In Eqs. (5.16) and (5.22), the electron mass should appear without the square, i.e., $m_{e}^{2}$ should be replaced by $m_{e}$. 